\title{
La responsabilité de l'Etat pour acte illicite, en situation de pandémie
}

\author{
Une approche prospective dans \\ le cadre des mesures de lutte contre \\ le COVID-19
}

La pandémie due au nouveau coronavirus a frappé la population avec une ampleur exceptionnelle. Afin de combattre la propagation de l'agent pathogène et de préserver les capacités du système de soins en Suisse, les autorités fédérales et cantonales ont adopté une politique sanitaire d'urgence, dans un contexte marqué par d'importantes incertitudes scientifiques. La gestion d'une telle crise donne lieu à des choix de mesures de prévention et de lutte, en fonction des connaissances disponibles. D'éventuels préjudices associés à ces mesures ou à leur insuffisance ouvrent la réflexion sur l'existence d'une responsabilité de l'Etat pour ses actes illicites. Celle-ci se place tant sur le terrain des mesures ordonnées que de l'omission illicite d'en prononcer ; dans ce second cas, l'autorité n'est pas supposée faire tout ce qui possible, mais uniquement ce qui est approprié et raisonnable.

I. Introduction

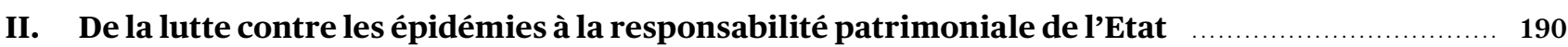

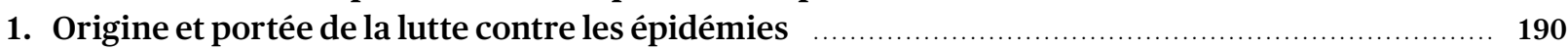

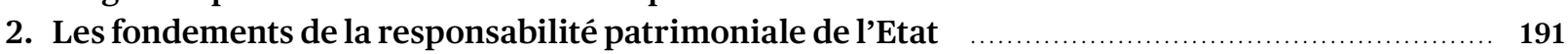

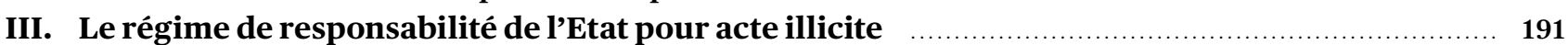

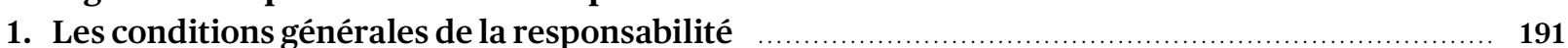

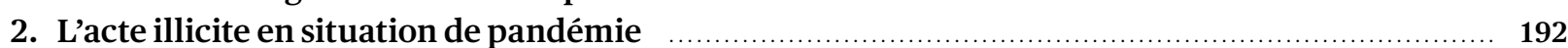

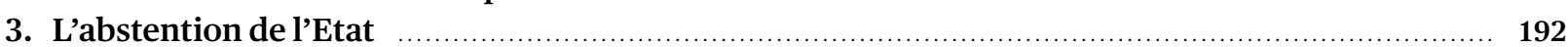

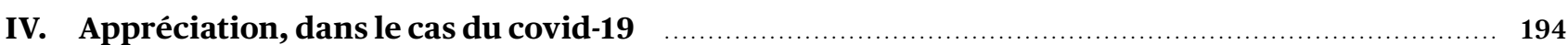

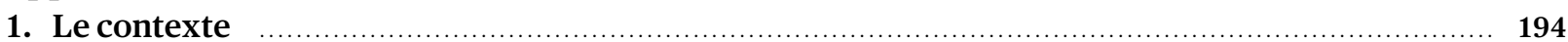

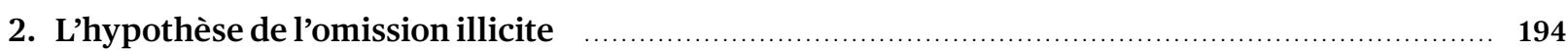

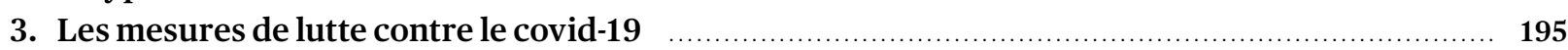

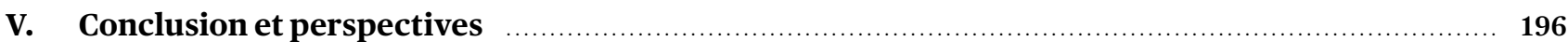

Citation:

THIERRY LARGEY, La responsabilité de l'Etat pour acte illicite, en situation de pandémie, sui generis 2021, p. 189

Thierry Largey, Professeur associé à l'Université de Lausanne. URL: sui-generis.ch/182

DOI: https://doi.org/10.21257/sg.182

Ce(tte) œuvre est mise à disposition selon les termes de la Licence Creative Commons Attribution - Partage dans les Mêmes Conditions 4.0 International. 


\section{Introduction}

1 La notion de pandémie est définie comme une «épidémie qui survient dans une vaste zone géographique affectant un grand nombre de pays», l'épidémie désignant quant à elle une «accumulation inhabituelle, limitée dans le temps et dans l'espace, d'une maladie dans une population ". ${ }^{1}$ Dans la législation suisse, les épidémies visent les maladies transmissibles et résultent d'infections. ${ }^{2}$

2 La Suisse s'est préparée à faire face à des pandémies en se dotant d'instruments juridiques permettant d'engager les mesures nécessaires à la protection de l'être humain contre les maladies transmissibles. ${ }^{3}$ La révision totale de la loi fédérale sur les épidémies (LEp) ${ }^{4}$ entrée en

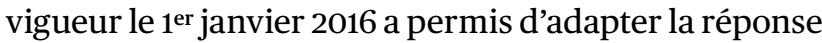
législative de la Confédération au risque accru de voir de nouvelles maladies causer des dommages massifs à l'échelle mondiale. ${ }^{5}$

3 La LEp a pour but de garantir que les mesures de prévention et de lutte soient mises sur pied en temps utile, conformément aux besoins et, selon la situation, de manière uniforme sur l'ensemble du territoire. ${ }^{6}$ Elle prévoit une action coordonnée de la Confédération et des cantons afin de prévenir et combattre l'apparition et la propagation des maladies transmissibles (art. 2 al. 1 LEp).

4 Le nouveau coronavirus (SARS-CoV-2, covid-19) est à l'origine d'une pandémie mondiale aux conséquences sanitaires, économiques et sociales inédites. Face à l'ampleur et l'intensité de cette pandémie, le choix des mesures à engager relève d'une complexité remarquable, aussi bien au regard de l'évaluation ex ante de leurs effets sur la situation sanitaire que de leurs conséquences sur les droits et les obligations des personnes. C'est dans un tel contexte que se pose la question d'une éventuelle responsabilité encourue par la Confédération et/ou les cantons.

5 La présente contribution tend à examiner le cadre de la responsabilité patrimoniale de l'Etat en situation de pandémie - à l'aune en particulier de la situation imposée par le covid-19. Le propos traite en premier lieu des fondements d'une éventuelle responsabilité étatique (II),

1 OFSP, Plan suisse de pandémie Influenza, Stratégies et mesures pour la préparation à une pandémie d'Influenza, Berne 2018, p. 124 s.

2 Message concernant la révision de la loi fédérale sur la lutte contre les maladies transmissibles de l'homme (Loi sur les épidémies, LEp) (FF 2011291), p.339. La législation sur les épidémies n’englobe ainsi pas les maladies qui ne sont pas provoquées par un agent pathogène.

3 Voir à ce propos FRÉDÉRIC BERNARD, La loi sur les épidémies à l'épreuve du nouveau coronavirus, Jusletter du 30 mars 2020.

4 Loi fédérale sur la lutte contre les maladies transmissibles de l'homme (Loi sur les épidémies, LEp; RS 818.101).

5 Message LEp (n. 2), p.302.

6 Message LEp (n. 2), p. 318. avant d'aborder les circonstances dans lesquelles celleci pourrait être reconnue. La matière étant vaste, la présente contribution est restreinte à la responsabilité de l'Etat pour acte illicite (III et IV).

\section{De la lutte contre les épidémies à la responsabilité patrimoniale de l'Etat}

\section{Origine et portée de la lutte contre les épidémies}

En vertu de l'art. 118 al. 2 let. b Cst., 7 la Confédération est 6 tenue de légiférer sur «la lutte contre les maladies transmissibles». Elle jouit à ce titre d'une compétence concurrente dotée d'un effet dérogatoire subséquent, ${ }^{8}$ concrétisée avec l'adoption de la LEp. Suivant l'art. 118 al. 1 Cst., la Confédération doit en outre prendre des mesures afin de protéger la santé. Elle doit non seulement définir le cadre et les principes de la lutte contre les épidémies et les pandémies, mais doit également prévoir et engager les actions et les moyens appropriés en vue de mettre en œuvre cet objectif.

La révision totale de la LEp initiée en 2012 avait pour but 7 de créer les conditions nécessaires propres à planifier des mesures plus cohérentes au niveau national sous la direction de la Confédération et à renforcer les mesures préventives qui s'avèrent les plus efficientes. ${ }^{9}$

L'art. 2 al.1LEp organise la lutte contre les maladies trans- 8 missibles en deux temps. Il s'agit en premier lieu de prévenir et combattre leur apparition. Dès lors qu'une épidémie s'est néanmoins déclenchée, il s'agit en second lieu de prévenir et combattre sa propagation. Les mesures propres à atteindre ce but doivent permettre de réduire la reproduction de l'agent pathogène en agissant sur ses hôtes et leur comportement, en réduisant notamment le risque d'exposition à la maladie. A cet égard, la LEp envisage trois catégories de mesures de lutte, ayant toutes pour conséquence de produire des effets contraignants sur la population. ${ }^{10}$ La première affecte les personnes, telle la mise en quarantaine ou le port du masque de protection (art.30 ss. LEp); la deuxième vise la population, comme la fermeture des commerces et des musées (art. $40 \mathrm{LEp}$ ); la dernière concerne le transport international de personnes (art. 41 ss. LEp).

\footnotetext{
7 Constitution fédérale de la Confédération suisse du 18 avril 1999 (Cst; RS101).

8 Message relatif à une nouvelle constitution fédérale (FF 1997 I1), p.339.

9 Message LEp (n. 2), p. 292.

10 Voir à ce propos BERNARD (n. 3), p. $7 \mathrm{ss}$.
} 


\section{Les fondements de la responsabilité patrimoniale de l'Etat}

a) Les conséquences dommageables des mesures de lutte contre les épidémies

9 Les mesures prises en vertu des art. 30 ss. LEp sont susceptibles d'empiéter sur les droits fondamentaux garantis par la Constitution fédérale : la liberté de mouvement et l'intégrité physique relevant de la liberté personnelle (art. 10 al. 2 Cst.) ou encore la liberté économique (art. 27 Cst.). Des restrictions aux droits sont néanmoins admissibles aux conditions de l'art. 36 Cst. ${ }^{11}$

Plus encore, les mesures de lutte contre les maladies transmissibles peuvent être sources de dommages affectant les personnes. Par dommage, il faut entendre la différence qui existe entre le montant du patrimoine avant et après la mise en œuvre de la mesure restrictive - en l'espèce, destinée à combattre l'épidémie ou la pandémie. ${ }^{12}$

11 Dans un tel contexte, mis en évidence avec une acuité particulière dans le cas de la pandémie au covid-19,13 se pose invariablement la question de l'éventuelle responsabilité patrimoniale encourue par l'Etat, à raison d'une politique sanitaire inadéquate. Cette question n'a, à ce jour, guère été traitée en Suisse ; ${ }^{14}$ le Tribunal fédéral n'a pas encore eu à se prononcer à ce propos. Elle émerge toutefois dans certains pays où sont mis en cause l'objectif poursuivi par le gouvernement, le retard pris dans les mesures de réponse adéquates ou le manque de préparation et de transparence dans la lutte contre la pandémie. ${ }^{15}$

\section{b) Les sources de la responsabilité de l'Etat}

12 Dès lors que l'acte dommageable appartient au droit public, l'Etat n'est responsable que si une base légale le prévoit (principe de la légalité). L'Etat détermine en effet lui-même les conséquences juridiques de ses activités publiques. ${ }^{16}$ Le fondement constitutionnel de la respon-

11 Message LEp (n. 2), p.366; BERNARD (n.3), p. 6. L’intérêt public jus tifiant des mesures restrictives pour la population repose notamment sur le droit à la vie consacré à l'art. 10 al. 1 Cst. ou le droit à la santé reconnu en tant que but social à l'art. 41 al. 1 let. b Cst. Voir à ce propos GREGOR T. CHATTON, in : Martenet/Dubey (édit.), Constitution fédérale, Commentaire romand, à paraître en juin 2021, Art. 41 N 39.

12 ATF 132 III 353 c. 4 ; ATF 129 III331. TOBIAS JAAG, Le système général du droit de la responsabilité de l'Etat, in : Favre/Martenet/Poltier (édit.), La responsabilité de l'Etat, Genève 2012, p. 27.

13 Voir par exemple Le Temps du 17 mars 2021 (A l’Université de Lausanne, des étudiants éprouvés par les sessions d'examens en ligne).

14 Voir néanmoins infra IV.3.

15 Voir notamment, à propos des Pays-Bas, Le Grand Continent du 28 juin 2020 (La responsabilité des États dans la gestion de l'épidémie du Covid-19 : droit international et droit néerlandais).

16 PIERRE MOOR / ETIENNE POLTIER, Droit administratif, Vol. II : Les actes administratif et leur contrôle, Berne 2011, p. 836 . sabilité de la Confédération figure à l'art.146 Cst., lequel est concrétisé dans la LRCF. ${ }^{17}$

La LRCF ne s'applique qu'aux tâches de droit public in- 13 combant aux personnes investies d'une fonction publique de la Confédération (art. 1 al.1 LRCF). Au niveau cantonal, chaque canton a adopté sa propre réglementation sur la responsabilité, laquelle reprend en général les principes développés au niveau fédéral. ${ }^{18}$

Tant en droit fédéral que cantonal, l'Etat ne saurait assu- 14 mer une obligation générale d'indemniser tout préjudice qui découlerait de ses activités ou de son inactivité. Sa responsabilité ne peut être mise en cause que dans certaines circonstances prévues par la loi. L'une d'entre elles est caractérisée par un régime de responsabilité fondé sur l'existence d'un acte illicite (art. 3 al. 1 LRCF, art. 1 al. 1 LRECA). ${ }^{19}$

\section{Le régime de responsabilité de l'Etat pour acte illicite}

\section{Les conditions générales de la responsabilité}

La législation sur les épidémies ne prévoit pas de régime 15 spécial de responsabilité de la Confédération ou des cantons, en présence d'un acte illicite. Par conséquent, le régime général de la responsabilité de l'Etat s'applique pleinement à ces situations (art. 3 al. 2 LRCF). 20

En sus de l'illicéité de l'activité étatique en cause, la LRCF, 16 de même que les lois cantonales, soumettent la reconnaissance de la responsabilité des autorités à plusieurs conditions cumulatives (art. 3 al. 1 LRCF, art. 1 al. 1 LRECA) : l'existence d'un dommage causé par un fonctionnaire dans l'exercice de ses fonctions. ${ }^{21}$ De surcroît, la responsabilité est admise indépendamment d'une faute commise par le fonctionnaire-à l'exception de certaines législations cantonales (Grisons, Lucerne, Genève). ${ }^{22}$

17 Loi fédérale sur la responsabilité de la Confédération, des membres de ses autorités et de ses fonctionnaires du 14 mars 1958 (LRCF; RS170.32).

18 Voir par exemple, dans le canton de Vaud, la loi sur la responsabilité del'Etat, des communes et de leurs agents (LRECA; RSVD 170.11).

19 Nous renonçons à examiner dans la présente contribution la responsabilité étatique à raison de ses actes licites. Infra $\mathrm{V}$.

20 FLORENCE AUBRY GIRARDIN, Responsabilité de l'Etat: un aperçu de la jurisprudence du Tribunal fédéral, in : Favre/Martenet/Poltier (édit.), La responsabilité de l'Etat, Genève 2012, p.114ss., avec les exemples cités.

21 JAAG (n. 12), p. 27; PIERRE TSCHANNEN / ULRICH ZIMMERLI/ MARKUS MÜLLER, Allgemeines Verwaltungsrecht, Berne 2014, p. 598.

22 MOOR/POLTIER (n. 16), p. 852. Dans le canton de Genève, l’art.1 al.1 LREC-GE (Loi sur la responsabilité de l'Etat et des communes du 24 février 1989; RSGE A 240) exige que l'acte illicite soit commis intentionnellement, par négligence ou par imprudence. 


\section{L'acte illicite en situation de pandémie}

17 S’agissant des épidémies et des pandémies, il paraît hautement improbable - voire d'emblée exclu dans le cas du covid-19-d'admettre une quelconque responsabilité étatique à raison de l'existence même de la maladie transmissible ou de sa propagation sur le territoire helvétique. Aucune responsabilité étatique absolue ne saurait être reconnue pour la seule existence de conséquences dommageables d'une épidémie sur la santé, la vie ou les activités économiques. L'Etat n'est pas responsable du nouveau coronavirus, mais uniquement de la manière dont il gère la crise sanitaire qui en découle et organise la lutte.

La responsabilité de l'Etat pour acte illicite n'est envisageable qu'en présence d'une mesure publique (acte par commission) ${ }^{23}$ de lutte contre la maladie prononcée par la Confédération ou les cantons qui peut être considérée comme illicite. La notion d'illicéité caractérise les actes jugés non conformes au droit; elle suppose que l'Etat, au travers de ses organes ou de ses agents, ait violé, sans motifs justificatifs, des prescriptions destinées à protéger un bien juridique. Dit autrement, un dommage n'est en principe indemnisable qu'en présence d'une atteinte à un droit protégé par l'ordre juridique. ${ }^{24}$ Selon la jurisprudence, l'illicéité englobe la violation de principes généraux du droit, telle l'obligation, pour celui qui crée une situation dangereuse, de prendre les mesures propres à prévenir un dommage. ${ }^{25} \mathrm{~A}$ contrario, un acte légitimé par une règle de droit ou les devoirs de service, sans qu'aucune faute n’a été commise dans l'exercice de l'activité, est licite. ${ }^{26}$

19 Dans une première hypothèse, lorsque l'acte dommageable consiste dans l'atteinte à un droit absolu comme la vie, la santé humaine ou le droit de propriété, l'illicéité est d'emblée réalisée. Il n'est alors pas nécessaire de rechercher si et de quelle manière l'auteur a violé une norme de comportement spécifique. On parle à cet égard d'illicéité de résultat (Erfolgsunrecht). Dans une seconde hypothèse, lorsque l'acte dommageable porte atteinte à un autre intérêt, notamment dans le cas du dommage purement patrimonial, l'illicéité n'est reconnue que s'il existe un «rapport d'illicéité». S'agissant de la responsabilité étatique, l'Etat doit avoir violé une norme de comportement-ayant pour but de protéger lebienjuridique-lepatrimoine-en

23 Un mouvement, une parole ou un écrit. Voir à ce propos ETIENNE POLTIER, La responsabilité de l'Etat pour acte illicite : l'exigence de l'illicéité, in : Favre/Martenet/Poltier (édit.), La responsabilité de l'Etat, Genève 2012, p. 60.

24 PIERRE WESSNER, Au menu: boeuf, salades et fromages contaminés ou la notion d'illicéité dans tous ses états, in : Aronovitz (édit.), Gastronomie, alimentation et droit, Mélanges en l'honneur de Pierre Widmer, Zurich 2003, N19.

25 ATF 132 II 305 c. 4.1 avec les références citées (affaire de la vache folle) 26 ATF 118 Ib 473 c. 2 =JdT 1994 I 650. cause (illicéité de comportement, Verhaltensunrecht);27 cette théorie se confond pratiquement avec celle de faute (objective). ${ }^{28}$ La violation doit revêtir en outre une certaine gravité. ${ }^{29}$

Dans le contexte de la pandémie du covid-19, la responsa- 20 bilité de l'Etat pourrait être recherchée dans deux situations. En premier lieu, le lésé pourrait invoquer une mesure ou un comportement de l'Etat ayant effectivement favorisé la diffusion de la maladie et sa contamination..$^{30}$ La question est alors de savoir si, en l'état des connaissances au moment du dommage, il y a motif à considérer que la Confédération ou les cantons ont créé ou favorisé une situation sanitaire dangereuse, par leurs actes. En second lieu, un administré pourrait s'en prendre à une mesure étatique de lutte contre la propagation de l'agent pathogène tenue pour illicite, dont il prétend être la victime. ${ }^{31}$ En toute hypothèse, quand bien même certaines mesures pourraient avoir été illicitement ordonnées, encore faudrait-il qu'il existe un rapport de causalité naturelle et adéquate entre l'acte en cause et l'atteinte à un droit protégé par la loi. ${ }^{32}$

\section{L'abstention de l'Etat}

\section{a) L'omission illicite}

La responsabilité de l'Etat est également susceptible 21 d'être engagée à la suite d'une abstention de sa part, lorsqu'est violée une obligation d'agir de sorte que l'omission apparaisse comme illicite. Cette abstention peut se traduire par une absence d'activité de l'Etat ou une activité de sa part jugée insuffisante ou inappropriée. On pourrait songer au retard à ordonner des mesures qui auraient permis de limiter la propagation de l'agent pathogène, à

27 ATF 132 II 305 c. 4.1; ATF 132 II 449 c. 3.3. JAAG (n. 12), p. 32; TOBIAS JAAG, Staats- und Beamtenhaftung, Bâle 2017, N104; TSCHANNEN/ ZIMMERLI/MÜLLER (n. 21), p. 606 ss; POLTIER (n. 23), p. 48, 57 s.; WESSNER (n. 24), N19; ULRICH HÄFELIN / GEORG MÜLLER / FELIX UHLMANN, Allgemeines Verwaltungsrecht, Zurich 2016, N2114 ss.

28 WESSNER (n. 24), $\mathrm{N} 27$ avec les références citées.

29 VINCENT MARTENET, La responsabilité de l'Etat en matière mobilière, in : Foëx/Hottelier (édit.), La garantie de la propriété à l'aube du XXIe siècle, Genève 2009, p. 55.

30 Tomberaient dans la catégorie des actes illicites les propos de l'Etat visant à ne pas porter de dispositif de protection dans un contexte où une telle mesure serait connue comme efficace contre la maladie. En revanche, tarder à la prononcer ou ne pas la dicter constitue une omission. Pour une autre situation, voir infra IV.3.

31 On songe davantage dans cette hypothèse aux conséquences économiques (purement patrimoniales) des restrictions -illicites, par exemple arbitraires ou disproportionnées - d'activités imposées à la population.

32 Voir à ce propos JAAG (n. 12), p. 34; TSCHANNEN/ZIMMERLI/MÜLLER (n. 21), p.602 s. A titre d'exemple, le Tribunal fédéral a reconnu que le recul de la consommation de certains produits et l'impossibilité de vendre ces derniers doivent être considérés comme une conséquence adéquate de la contamination de ces produits par les radiations atomiques issues du réacteur de Tchernobyl (ATF 116 II 480 c. $3 \mathrm{~b}=\mathrm{JdT} 1993$ I 19). 
l'éventuel manque de diligence dans le prononcé de mesures de soutien fondées sur l'art. 2 al. 2 let. fLEp, à une organisation déficiente au regard des exigences de l'art. 2 al. 2 let. d LEp ou encore à l'insuffisance de moyens de protection contre la maladie en violation de l'art. 2 al. 2 let. eLEp.

Dans l'affaire de la «vache folle» survenue dans les années 90, les faits tenus pour illicites consistaient tous en des omissions. La Confédération n'aurait pas pris suffisamment tôt un certain nombre de mesures d'interdiction propres à empêcher la chute des prix du bétail de boucherie et d'élevage. Dans une telle situation, ce n'est toutefois que si l'Etat était tenu de prendre les mesures prétendument omises et si cette obligation avait notamment pour but de protéger la valeur économique du bétail que sa responsabilité pouvait être engagée. ${ }^{33}$

23 Une omission peut être ainsi constitutive d'une illicéité, à condition qu'il existât, au moment déterminant, une norme juridique (norme de comportement) «qui sanctionnait explicitement l'omission commise ou qui imposait à l'Etat de prendre en faveur du lésé la mesure omise; un tel chef de responsabilité suppose donc que l'Etat ait eu une position de garant vis-à-vis du lésé et que les prescriptions qui déterminent la nature et l'étendue de ce devoir aient été violées ».34 Il s'agit d'établir à quelles conditions légales et constitutionnelles objectives le fait de ne pas agir était régulier (omission licite) ou irrégulier (omission illicite), en considérant la portée des compétences discrétionnaires de l'autorité.

En présence d'une abstention d'agir s'applique par conséquent la théorie de l'illicéité de comportement (Verhaltensunrecht), quel que soit le bien juridique digne de protection en cause. ${ }^{35}$ La norme de comportement doit être recherchée dans tout l'ordre juridique, y compris dans le droit non écrit. ${ }^{36}$

Dans l'affaire de la vache folle, le Tribunal fédéral a admis que la législation sur les épizooties avait non seulement pour objectifs la poursuite de motifs d'ordre sanitaire, mais également la protection des agriculteurs contre les atteintes susceptibles de leur causer un dommage de nature patrimoniale. Il a reconnu en l'art. $9 \mathrm{LFE}^{37}$ une norme de comportement. ${ }^{38}$

\footnotetext{
33 ATF 132 II 305 c. 4.2

34 ATF 132 II 305 c. 4.1. JAAG (n. 27), N78, 98.

35 POLTIER (n. 23), p. 60.

36 TSCHANNEN/ZIMMERLI/MÜLLER (n. 21), p. 610.

37 Loi fédérale sur les épizooties du 1er juillet 1966 (LFE; RS 916.40).

38 ATF 132 II 305 c. 4.2 .
}

\section{b) L'examen rétrospectif de l'obligation d'agir}

Même dans une position de garant, l'Etat n'est pas de 26 tenu de tout faire (quoi qu'il en coûte) pour prévenir le dommage; il lui incombe d'engager seulement les mesures raisonnables et appropriées à la situation, telles qu'elles ressortent des exigences exprimées dans la norme de comportement et des besoins de protection des administrés. ${ }^{39}$

La portée de l'obligation d'agir s'examine en fonction de 27 la situation régnant au moment où les omissions prétendument illicites se seraient produites, ${ }^{40}$ en particulier sur le plan des connaissances scientifiques. Il s'agit ainsi de procéder à un examen rétrospectif de la situation, impliquant de faire abstraction de ce qui est connu de l'épidémie et de ses conséquences au jour de l'examen de la responsabilité. En d'autres termes, il convient de se replacer dans le contexte de l'époque.

L'examen doit également tenir compte des difficultés 28 propres à la gestion d'une crise sanitaire. ${ }^{41}$ On peut à ce titre citer l'exigence de suivre l'évolution de l'épidémie en Suisse et à l'étranger, d'appliquer les recommandations des organismes internationaux comme l'OMS, de prendre en considération les inconnues scientifiques et les nouvelles connaissances acquises (en distinguant les informations consolidées de celles qui sont peu probantes ou partielles) ou encore la nécessité d'agir dans l'urgence sans disposer des moyens de pouvoir apprécier de manière exacte et complète les circonstances. De surcroît, il convient de prendre en considération le fait que l'Etat est tenu de veiller à ce que les mesures envisagées trouvent une certaine acceptation dans la population - de sorteà favoriser leur mise en œuvre et leur succès. Un tel impératif suppose du temps et on peut considérer que plus les mesures envisagées sont de nature à porter gravement atteinte à des libertés fondamentales ou des intérêts importants, plus elles sont susceptibles de requérir du temps de préparation et de mise en œuvre.

Selon le Tribunal fédéral, il découle de ces différentes 29 contingences que l'on ne saurait exiger de l'Etat qu'il prenne en toute occasion les meilleures décisions au meilleur moment, car cela reviendrait à le placer dans une situation quasi impossible. En effet, quoi qu'il puisse faire ou s'abstenir de faire, il serait exposé au risque d'être toujours responsable, soit d'avoir agi trop tard ou trop mollement, soit d'avoir agi trop tôt ou de manière trop incisive.

39 TSCHANNEN/ZIMMERLI/MÜLLER (n. 21), p. 610; POLTIER (n. 23), p. 58. 40 JAAG (n. 27), N100. Voir sur la question de la temporalité de l'intervention étatique ATF 132 II 449 c. 7.1.1.

41 ATF 132 II 305 c. 4.4; ATF 118 Ib 473 c. 7 =JdT 1994 I 650. Voir également ATF 131 II 670 c. 2.3 (affaire du SRAS). 
A ce titre, l'autorité dispose d'une certaine liberté d'appréciation, aussi bien quant au choix des mesures à prendre que du moment où il convient de les prononcer. Les mesures qui, au vu de l'ensemble des circonstances, n'excèdent pas ou n'abusent pas de la marge de manœuvre de l'autoritén'engagent pas la responsabilité del'Etat - quand bien même elles s'avèreraient a posteriori insuffisantes. ${ }^{42}$

\section{Appréciation, dans le cas du covid-19}

\section{Le contexte}

Le nouveau coronavirus a été détecté pour la première fois en Suisse en février 2020. Il s'est propagé avec une rapidité et une intensité telles que la Confédération a dû réagir dans l'urgence - en disposant d'un minimum d'informations et de connaissances scientifiques. ${ }^{43}$ La gestion de la crise sanitaire est par conséquent susceptible d'être exposée à la critique, l'expérience et le recul montrant que l'on aurait pu faire mieux ou différemment. Que l'on songe au port du masque de protection qui n'a été rendu obligatoire que tardivement, en octobre 2020 dans tous les espaces publics clos. L'omission de prononcer à temps des mesures qui se révèlent $a$ posteriori aptes à freiner la propagation du virus peut-elle conduire à reconnaître une responsabilité de la Confédération ou des cantons?

31 Le sujet est activement débattu en France où une possible responsabilité pour faute de l'Etat est envisagée. A l'instar de ce que la jurisprudence suisse a développé dans le cadre de la crise de la vache folle, le juge administratif français doit toutefois tenir compte des contraintes qui pèsent sur l'administration et ne peut exiger que l'autorité soit omnisciente ni qu'elle prenne des précautions démesurées. ${ }^{44}$

\section{L'hypothèse de l'omission illicite}

Reconnaître une omission illicite de l'Etat à raison de mesures de lutte contre le covid-19 exige que l'autorité se trouve dans une position de garant ${ }^{45}$ et ait violé ses obligations de manière manifeste. ${ }^{46} \mathrm{~A}$ ce titre, il n'existe pas d'obligation légale générale d'agir dans l'intérêt d'autrui. ${ }^{47}$

\footnotetext{
42 ATF 132 II 305 c. 4.4.

43 Voir à ce propos FRÉDÉRIC BERNARD, Lutte contre le nouveau coronavirus et respect des droits fondamentaux, Sécurité \& Droit 3/2020, p. 130.

44 ANNE JACQUEMET-GAUCHÉ, Coronavirus-Pénurie de masques: une responsabilité pour faute de l'État?, La Semaine Juridique 13/2020.

45 Supra III.3.a).

46 MARTENET (n. 29), p. 58.

47 Arrêt du Tribunal fédéral 2C_1059/2014 du 25 mai 2016 c. 5.3.
}

Dans l'affaire de la vache folle, le Tribunal fédéral a établi 33 que l'art. 9 LFE engage les autorités compétentes à agir, même en cas d'incertitude scientifique; il suffit que les mesures envisagées «paraissent propres » à empêcher l'apparition ou la propagation d'une épizootie d'après l'état de la science et de l'expérience. Par conséquent, la Confédération se trouvait dans une position de garant, sa responsabilité étatique pouvant être engagée en cas de violation de l'obligation d'agir. ${ }^{48}$

Dans les affaires de la listériose et de la vache folle en- 34 core, le Tribunal fédéral a examiné si les dispositions de l'ancienne LEp constituaient des normes de comportement - dans le cadre d'informations ayant causés des dommages d'ordre économique. Il arrive au constat que les dispositions dela législation sur les épidémies - comme celles de la législation sur les denrées alimentaires - visaient en premier lieu à protéger la vie et la santé de la population, et non pas d'autres intérêts de tiers, notamment d'ordre économique. Toutefois, dès lors que l'autorité est tenue de prendre en considération les intérêts privés dignes de protection lorsqu'elle prononce une mesure, l'aLEp avait aussi un effet protecteur pour tous les particuliers qui sont concernés par la mesure en cause. ${ }^{49}$

La révision totale de la LEp en 2016 ne modifie en rien ce 35 constat qui consacre le caractère protecteur de la législation sur les épidémies. La loi enjoint l'Etat d'adopter un comportement propre à prévenir les dommages aux droits absolus que sont la vie et la santé, mais également les préjudices purement économiques qui résultent des maladies transmissibles. La portée élargie de la protection apparaît expressément dans la nouvelle loi. Conformément à l'art. 2 al. 2 let. fLEp, les mesures de lutte des art. 30 ss. LEp ont pour objectif de « réduire les effets des maladies transmissibles sur la société et les personnes concernées ». Sont concernés non seulement les effets sur la santé des personnes, mais également les effets sur d'autres volets sociaux, notamment les processus économiques et la vie en société en général.50

Dans le cas du coronavirus, une omission des autorités 36 pourrait en particulier être invoquée à propos de l'indisponibilité des masques en début de pandémie, étant reproché une impréparation et l'engagement insuffisant de mesures de protection des individus. De même pourrait être invoqué le retard de la vaccination ayant conduit

\footnotetext{
48 ATF 132 II 305 c. 4.2 et 4.3; ATF 126 II 63 c. 3a. Voir également WESSNER (n. 24), N25 et 31. Voir en outre l'art. 2 let. bLFE.

49 ATF 118 Ib 473 c. 5 = JdT 1994 I 650; ATF 126 II 63 c. 3a; Arrêt du Tribunal fédéral 2A_276/1989 du 13 novembre 1992 c.2d (non publié). POLTIER (n.23), p. 58.

50 Message LEp (n. 2), p. 339.
} 
à un surcroît de contaminations, en tant qu'omission d'agir à temps. ${ }^{51}$

La seule omission de l'Etat à prévenir et combattre la propagation d'une maladie infectieuse ne suffit pas encore à engager sa responsabilité. Du point de vue méthodologique, s'impose l'examen rétrospectif de l'obligation d'agir de la collectivité publique - plus précisément de l'obligation qui lui incombait de prendre les mesures qu'on lui reproche d'avoir omises, afin d'empêcher l'apparition ou la propagation de l'épidémie (art. 2 al. 1 et 2). Doivent être prises en considération l'ensemble des circonstances replacées dans le contexte du moment déterminant. De surcroît, les mesures omises doivent être raisonnables et appropriées au besoin de protection du lésé. ${ }^{22}$ S’il est établi, au terme de cet examen, quel'Etat avait violé son obligation d'agir, son omission est à considérer comme illicite.

Dans l'hypothèse d'une omission avérée lors de la campagne de vaccination, la situation doit être examinée en fonction des circonstances qui prévalaient au moment où le lésé prétend à l'inaction dommageable de l'Etat. Il s’agit notamment de tenir compte des autorisations délivrées par Swissmedic, des livraisons effectuées par les fournisseurs, des stocks disponibles ou encore des difficultés logistiques à organiser la vaccination dans un contexte unique et exceptionnel. Une omission illicite ne peut alors guère être reconnue qu'en présence d'une désorganisation manifeste ou d'une impréparation évidente conduisant à des retards injustifiés et déraisonnables. S’agissant du port du masque, l'omission devrait être considérée comme illicite si les autorités avaient tardé à l'imposer, non par manque de connaissances de ses effets protecteurs, mais par une insuffisance de masques disponibles consécutive à une gestion manifestement déficiente de l'approvisionnement - pour autant que d'autres mesures n'aient pas été engagées de sorte à pallier le manque de masques, l'Etat disposant à cet égard d'un large pouvoir d'appréciation quant aux mesures de prévention et de lutte à ordonner.

39 Pour que la responsabilité de l'Etat soit engagée, encore faut-il que l'omission illicite se trouve dans un rapport de causalité naturelle et adéquate avec le dommage - la causalité étant hypothétique en présence d'une omission. ${ }^{53}$ La question du lien de causalité ne peut alors être résolue indépendamment du manquement de l'autorité étatique

\footnotetext{
51 A noter que les dommages qui font suite à une vaccination recom mandée ou obligatoire sont couverts par l'art. 68 LEp en tant que conséquences d'actes licites.

52 Supra III.3.b).

53 Arrêt du Tribunal administrative fédéral 2008/6 du 17 janvier 2008 c. 4.2.2. JAAG (n. 27), N144.
}

et de l'éventuelle faute du lésé. ${ }^{54}$ A cet égard, la causalité est absente lorsque la mesure omise apparaît rétrospectivement inapte (inappropriée) à empêcher-pour le moins à empêcher en grande partie - le dommage de survenir.

Dans l'hypothèse où une omission illicite concernant le 40 port du masque ou la vaccination devait être admise, le lésé doit encore démontrer un rapport de causalité hypothétique entre l'abstention et le dommage - en établir la vraisemblance prépondérante (" eine überwiegende Wahrscheinlichkeit»). ${ }^{55}$ Dans le cours ordinaire des choses et l'expérience générale de la vie, la personne contaminée ne l'aurait-elle pas été si elle avait porté un masque alors qu'il n'était pas prescrit ou si elle avait été vaccinée à temps - compte tenu également des autres mesures de prévention ordonnées? Les incertitudes quant aux circonstances individuelles de la contamination ou à l'immunité apportée par le vaccin rendent un tel lien périlleux à établir. En outre, le lésé ne doit pas avoir interrompu le lien de causalité par un comportement personnel fautif l'exposant au virus, en ne respectant pas d'autres règles de prévention notamment (distanciation sociale, restrictions d'activités et de rassemblement, ....). ${ }^{56}$

\section{Les mesures de lutte contre le covid-19}

En avril 2021, moins d'une dizaine d'actions en responsa- 41 bilité de la Confédération étaient déposées auprès du DFF, dont celle de la Fédération suisse des centres de fitness. Celle-ci prétend que la fermeture des centres de fitness "provoque d'importantes pertes de chiffre d'affaires qu’elle juge disproportionnées ». ${ }^{57}$

La responsabilité de l'Etat ne peut être engagée que s'il 42 est établi par le lésé que la mesure litigieuse (acte par commission) est illicite. Deux aspects méritent d'être à cet égard considérés.

D’une part, la législation sur les épidémies confère un 43 large pouvoir d'appréciation aux cantons et à la Confédération, s'agissant de mesures de lutte à ordonner. Selon les circonstances, un excès ou un abus du pouvoir d'appréciation conféré par la loi peut néanmoins être constitutif d'illicéité ; 58 l'action en responsabilité ne saurait toutefois priver l'autorité de sa marge de manœuvre. ${ }^{59}$ L’art. 40 al. 2 let. b. LEp mentionne, parmi les mesures

54 Arrêt du Tribunal fédéral 2C_1059/2014 du 25 mai 2016 c. 5.3. 55 ATF 141 V 51 c. 8.1.

56 JAAG (n. 27), N150 s. Arrêt du Tribunal fédéral 2C_4/2000 du 3 juillet 2003 c. 8.5 .

57 Le Temps du 4 avril 2021 (Les fitness déposent une plainte contre la Confédération).

58 ATF 132 II 485 c. 3.2; ATF 118 Ib 473 c. 2=JdT 1994 I 650 (affaire de la listériose).

59 MARTENET (n.29), p. 59. 
visant la population ou certains groupes de personnes, la fermeture des entreprises privées, dont font partie les fitness. En y ayant recours, l'autorité n'excède ainsi pas son pouvoir d'appréciation. Elle pourrait néanmoins en abuser si la fermeture ordonnée devait être considérée comme disproportionnée ou abusive (ce que prétend la Fédération). L'art. 40 al. 1 et 3 LEp limite l'activité de l'autorité aux seules mesures "nécessaires ", tant à propos de leur intensité que de leur durée. Cette condition est à examiner à l'aune de l'ensemble des circonstances; il convient notamment de prendre en considération les incertitudes qui subsistent et les contraintes qui pèsent sur l'autorité.

D'autre part, l'évolution des connaissances de la maladie transmissible a des répercussions sur le choix des mesures de lutte à ordonner. L'autorité est tenue d'adapter le régime de mesures aux progrès des connaissances. ${ }^{60}$ En substance, si une mesure devient subséquemment disproportionnée, elle doit être abandonnée ou remplacée lorsqu'elle ne permet plus, ou pas suffisamment d'atteindre le but de protection (maxime de l'aptitude); elle doit être allégée ou modifiée lorsqu'il est possible de le faire sans remettre en cause l'objectif visé (maxime de la nécessité). A cet effet, les mesures doivent être réexaminées régulièrement (art. 40 al. 3 LEp) - l'autorité disposant en la matière d'une liberté d'appréciation.

Lorsque le lésé invoque une perte de chiffre d'affaires, autrement dit un préjudice purement patrimonial, il doit alléguer une norme de comportement ayant pour but de protéger le revenu de son activité professionnelle. On l'a vu, la LEp a pour objectif de protéger la vie et la santé, mais également les processus économiques. Les mesures ordonnées doivent réduire les effets des maladies transmissibles sur la société (art. 2 al. 2 let. fLEp), non pas les accroître - hormis lorsque cela est rendu «nécessaire» (art.1LEp) par la protection de biens juridiques prépondérants comme la santé publique. Dans le cas où l'existence d'un acte illicite ne peut être exclue, le lésé doit en outre démontrer un lien de causalité adéquate, ainsi que le dommage qu'il a subi.

60 ATF 132 II 305 c. 4.1

\section{Conclusion et perspectives}

Le Tribunal fédéral n’a pas eu, jusqu’à présent, à se pro- 46 noncer sur une potentielle activité illicite de l'Etat dans la gestion de la pandémie du covid-19. S'il le devait, ce serait notamment à raison d'une abstention d'agir supposée des autorités. Selon la jurisprudence antérieure à la révision totale de la LEp, la Confédération et les cantons ont une position de garant en situation d'épidémie ou de pandémie. Dans le droit actuel, l'art. 2 LEp - avec les art.30 ss LEp - constitue une norme de comportement qui leur impose de prendre les mesures aptes à protéger la population contre les effets dommageables des maladies transmissibles. Il ne peut toutefois pas être attendu de l'autorité qu'elle fasse rétrospectivement tout juste, mais seulement qu'elle engage une activité adéquate en fonction des circonstances et des connaissances au moment des faits.

La responsabilité de l'Etat pourrait être-prioritairement 47 même - envisagée dans un autre cadre, celui de ses actes licites. On peut à cet égard songer à la fermeture conforme au droit des commerces, des restaurants ou encore des musées ayant pour conséquences des pertes financières considérables. L’art. 146 Cst., pas plus que la LRCF, ne prévoit d'indemnisation dans de telles situations; ils nel'excluent toutefois pas, au regard notamment d'une interprétation conforme au principe d'égalité de l'art. 8 Cst. ${ }^{61}$ Au niveau cantonal, plusieurs cantons ont adopté des dispositions générales ou limitatives de responsabilité pour certains actes licites de l'Etat. Une telle responsabilité relève toutefois d'un domaine de recherche en soi que nous réservons à des développements ultérieurs.

61 THIERRY TANQUEREL, La responsabilité de l'Etat pour acte licite, in : Favre/Martenet/Poltier (édit.), La responsabilité de l'Etat, Genève 2012, p. 93. 